\title{
Application of variable interchange method for solution of two-dimensional Burgers' equations
}

\author{
Vildan Gulkac \\ Mathematics Department, Faculty of Science and Arts, Kocaeli University, Kocaeli, Turkey.
}

Received: 26 January 2016, Accepted: 4 March 2016

Published online: 11 February 2017.

\begin{abstract}
In this paper, the change-of-variable method introduced by Boadways [1] is presented for solving a two-dimensional Burgers' equation. The results are compared with those obtained earlier by other authors $[3,6,9]$.
\end{abstract}

Keywords: Burger's equations, Boadways transformation.

\section{Introduction}

Burgers' equation has been fundamental partial differential equation from fluid mechanics. Systems of this partial differential equations have attracted much attention in studying evolution equations describing turbulence, the approximate theory of flow thorough a shock wave traveling in a viscous fluid. Fletcher using the Hope-Cole transformation [2] gave an analytic solution of two dimensional Burgers' equations. The numerical solutions of this equations have been studied by several authors. Jain and Holla [3] developed two algorithms based on cubic spline function method and Goyon [4] used several multilevel schemes with ADI, Wubs and Goede [5] have applied an expilicit-implicit method, Bahadır [6] have applied implicit finite-difference method, El-Sayed and Kaya [7] and Zhu et all. [8] have applied Adomian decomposition method, Srivastava et all. [9] have applied a Crank-Nicolson method.

In this study, we proposed a relatively simple numerical method for solution two-dimensional Burgers' equation by independent variable interchange which is an extension as well as modification of Boadway's transformation [10,11,12].

\section{Two-dimensional Burgers' equations}

Consider the following system of two-dimensional Burgers' equations taken from [6].

$$
\begin{aligned}
& \frac{\partial u}{\partial t}=\frac{1}{R}\left(\frac{\partial^{2} u}{\partial x^{2}}+\frac{\partial^{2} u}{\partial y^{2}}\right)-u \frac{\partial u}{\partial x}-v \frac{\partial u}{\partial y} \\
& \frac{\partial v}{\partial t}=\frac{1}{R}\left(\frac{\partial^{2} v}{\partial x^{2}}+\frac{\partial^{2} v}{\partial y^{2}}\right)-u \frac{\partial v}{\partial x}-v \frac{\partial v}{\partial y}
\end{aligned}
$$

subject to the conditions

$$
\begin{aligned}
& u(x, y, 0)=f(x, y),(x, y) \in D \\
& v(x, y, 0)=g(x, y),(x, y) \in D
\end{aligned}
$$

and

$$
u(x, y, t)=f_{1}(x, y, t),(x, y) \in \partial D, t>0
$$




$$
v(x, y, t)=g_{1}(x, y, t),(x, y) \in \partial D, t>0
$$

Here $D=\{(x, y): a \leq x \leq b, a \leq y \leq b\}, \partial D$ denotes the boundary of $\mathrm{D}, u(x, y, t)$ and $v(x, y, t)$ are the velocity components to be determined $f, g, f_{1}, f_{2}$ are known functions and $\mathrm{R}$ is the Reynolds number.

\section{Boadway's transformation}

To solve eqns. (1) and (2), according to the extension of Boadway's Transformation, for the purpose of illustration, the governing equations are presented for a two-dimensional case. Hence, the particular case, of the curvilinear transformation for the two-dimensional Burger's equations can be performed, for example, following Boadway's treatment of fluid-flow problems. The wave function my be defined as a function of $x, y$, i.e. [10].

$$
u=u(x, y)
$$

and hence,

$$
d u=\frac{\partial u}{\partial x} d x+\frac{\partial u}{\partial y} d y
$$

one may introduce a new dumy variable $M=M(x, y)$ and

$$
\partial M=\frac{\partial M}{\partial x} d x+\frac{\partial M}{\partial y} d y
$$

what is the wanted are new functions,

$$
x=x(M, u)
$$

and $y=y(M, u)$

then

$$
\begin{aligned}
& d x=\frac{\partial x}{\partial M} d M+\frac{\partial x}{\partial u} d u \\
& d y=\frac{\partial y}{\partial M} d M+\frac{\partial y}{\partial u} d u
\end{aligned}
$$

$$
\begin{aligned}
& d x=\frac{\partial x}{\partial M}\left[\frac{\partial M}{\partial x} d x+\frac{\partial M}{\partial y} d y\right]+\frac{\partial x}{\partial u}\left[\frac{\partial u}{\partial x} d x+\frac{\partial u}{\partial y} d y\right] \\
& d y=\frac{\partial y}{\partial M}\left[\frac{\partial M}{\partial x} d x+\frac{\partial M}{\partial y} d y\right]+\frac{\partial y}{\partial u}\left[\frac{\partial u}{\partial x} d x+\frac{\partial u}{\partial y} d y\right]
\end{aligned}
$$

from which

$$
\frac{\partial M}{\partial x}=\frac{\frac{\partial y}{\partial u}}{B}
$$

and $\frac{\partial u}{\partial x}=\frac{\frac{-\partial y}{\partial M}}{B}$ where B is the determinant of the matrix. Similarly collecting dy terms, we obtain

$$
\frac{\partial M}{\partial y}=\frac{\frac{\partial y}{\partial u}}{B}
$$

and $\frac{\partial u}{\partial y}=\frac{\frac{\partial x}{\partial M}}{B}$ from which in dealing with second derivatives, we obtain

$$
\frac{\partial^{2} u}{\partial x^{2}}=\frac{1}{B^{3}}\left[\frac{\partial y}{\partial u}\left(\frac{\partial y}{\partial M} \frac{\partial B}{\partial M}-B \frac{\partial^{2} y}{\partial M^{2}}\right)-\frac{\partial y}{\partial M}\left(\frac{\partial y}{\partial M} \frac{\partial B}{\partial u}-B \frac{\partial^{2} y}{\partial M \partial u}\right)\right]
$$

(c) 2017 BISKA Bilisim Technology 
similarly,

$$
\frac{\partial^{2} u}{\partial y^{2}}=\frac{1}{B^{3}}\left[\frac{\partial x}{\partial u}\left(\frac{\partial x}{\partial M} \frac{\partial B}{\partial M}-B \frac{\partial^{2} x}{\partial M^{2}}\right)-\frac{\partial x}{\partial M}\left(\frac{\partial x}{\partial M} \frac{\partial B}{\partial u}-B \frac{\partial^{2} u}{\partial M \partial u}\right)\right]
$$

If one lets $M=x$ then

$$
\begin{gathered}
\frac{\partial x}{\partial M}=1, \frac{\partial x}{\partial u}=0, \frac{\partial y}{\partial M}=\frac{\partial y}{\partial x}, B=\frac{\partial y}{\partial u}, \frac{\partial B}{\partial M}=\frac{\partial^{2} y}{\partial M \partial u}, \frac{\partial B}{\partial M}=\frac{\partial^{2} y}{\partial u^{2}} \\
\frac{\partial^{2} u}{\partial x^{2}}=\frac{1}{\left(\frac{\partial y}{\partial u}\right)^{3}}\left[\frac{\partial y}{\partial u}\left(\frac{\partial y}{\partial u} \frac{\partial^{2} y}{\partial u \partial x}-\frac{\partial y}{\partial u} \frac{\partial^{2} y}{\partial x^{2}}\right)-\frac{\partial y}{\partial x}\left(\frac{\partial y}{\partial x} \frac{\partial^{2} y}{\partial u^{2}}-\frac{\partial y}{\partial u} \frac{\partial^{2} y}{\partial x \partial u}\right)\right] \\
\frac{\partial^{2} u}{\partial y^{2}}=\frac{1}{\left(\frac{\partial y}{\partial u}\right)^{3}}\left[-\frac{\partial^{2} y}{\partial u^{2}}\right]
\end{gathered}
$$

\section{Transformation of Burgers' equations}

The transformed eqn. (1), (2), expressing $y$ as dependent and $u, v, x, t$ as independent variables following Gulkac [10], can be written as

$$
\frac{\partial y}{\partial t}=-\frac{1}{R}\left\{\frac{\partial^{2} y}{\partial x^{2}}\left(\frac{\partial y}{\partial u}\right)^{3}+\frac{\partial^{2} y}{\partial u^{2}} \frac{\partial y}{\partial u}\left[1+\left(\frac{\partial y}{\partial x}\right)^{2}\right]-2 \frac{\partial^{2} y}{\partial x \partial u} \frac{\partial y}{\partial x}\left(\frac{\partial y}{\partial u}\right)^{2}\right\}+u \frac{\partial y}{\partial x}+v
$$

similarly eqn.(2) written as

$$
\frac{\partial y}{\partial t}=-\frac{1}{R}\left\{\frac{\partial^{2} y}{\partial x^{2}}\left(\frac{\partial y}{\partial v}\right)^{3}+\frac{\partial^{2} y}{\partial v^{2}} \frac{\partial y}{\partial v}\left[1+\left(\frac{\partial y}{\partial x}\right)^{2}\right]-2 \frac{\partial^{2} y}{\partial x \partial v} \frac{\partial y}{\partial x}\left(\frac{\partial y}{\partial v}\right)^{2}\right\}+u \frac{\partial y}{\partial x}+v
$$

and new domain and boundary of $\mathrm{D}$ can be written as

$$
\begin{aligned}
& y(x, u, 0)=f(x, u),(x, u) \in D \\
& y(x, v, 0)=g(x, v),(x, v) \in D
\end{aligned}
$$

and

$$
\begin{aligned}
& y(x, u, t)=f_{1}(x, u, t), x, u \in \partial D, t>0 \\
& y(x, v, t)=g_{1}(x, v, t), x, v \in \partial D, t>0
\end{aligned}
$$

where

$$
D=\{(x, u),(x, v): a \leq x \leq b, a \leq u, v \leq b\}
$$

\section{Discretization of transformed equation}

In this study, on the x-u grid and x-v grid, and choosing $\Delta u, \Delta v$ such that $u_{j}=u_{0}+j \Delta u, v_{j}=v_{0}+j \Delta v, j=0,1, \ldots, N$, $\left(u_{0}=v_{0}=0\right)$.

Assume that at some instant of time $t=t_{m}=m \Delta t$, the total number of velocity components and their locations are denoted by $y_{i, j}^{m}$. We evaluate the values of $y_{i, j}^{m}$ on this grid at successive time steps $t_{m}=t_{0}+m \Delta t, t_{0}$ is the time when numerical computations are commenced and $\Delta t$ is the time interval. We also point out $u_{0}+k \Delta u$ and $v_{0}+k \Delta v$ are the $\mathrm{k}+\mathrm{h}$ velocity components, on which the velocity components are $u_{k}$ and $v_{k}$ in the $(x, y)$ plane. Therefore, we can write 
equation (3) as equation (5)

$$
\begin{aligned}
& \frac{y_{i, j}^{n+1}-y_{i, j}^{n}}{\Delta t}=\frac{1}{R}\left\{-\frac{y_{i+1, j}^{n}-2 y_{i, j}^{n}+y_{i-1, j}^{n}}{\Delta x^{2}}\left(\frac{y_{i, j+1}^{n}-y_{i, j-1}^{n}}{2 \Delta u}\right)^{3}-\frac{y_{i, j+1}^{n}-2 y_{i, j}^{n}+y_{i, j-1}^{n}}{\Delta u^{2}} \frac{y_{i, j}^{n}-y_{i, j-1}^{n}}{\Delta u}\left[1+\left(\frac{y_{i+1, j}^{n}-y_{i, j}^{n}}{\Delta x}\right)^{2}\right]\right. \\
& \left.+2 \frac{y_{i+1, j+1}^{n}-y_{i+1, j-1}^{n}-y_{i-1, j+1}^{n}+y_{i-1, j-1}^{n}}{\Delta x \Delta u} \frac{y_{i+1, j}^{n}-y_{i, j}^{n}}{\Delta x}\left(\frac{y_{i, j+1}^{n}-y_{i, j}^{n}}{\Delta u}\right)^{2}\right\}+u_{i, j}^{n-1} \frac{y_{i+1, j}^{n}-y_{i-1, j}^{n}}{2 \Delta x}-v_{i, j}^{n-1}
\end{aligned}
$$

similarly, the finite-difference equation of (4) can be written as (6)

$$
\begin{aligned}
& \frac{y_{i, j}^{n+1}-y_{i, j}^{n}}{\Delta t}=\frac{1}{R}\left\{-\frac{y_{i+1, j}^{n}-2 y_{i, j}^{n}+y_{i-1, j}^{n}}{\Delta x^{2}}\left(\frac{y_{i, j+1}^{n}-y_{i, j-1}^{n}}{2 \Delta v}\right)^{3}-\frac{y_{i, j+1}^{n}-2 y_{i, j}^{n}+y_{i, j-1}^{n}}{\Delta v^{2}} \frac{y_{i, j}^{n}-y_{i, j-1}^{n}}{\Delta v}\left[1+\left(\frac{y_{i+1, j}^{n}-y_{i, j}^{n}}{\Delta x}\right)^{2}\right]\right. \\
& \left.+2 \frac{y_{i+1, j+1}^{n}-y_{i+1, j-1}^{n}-y_{i-1, j+1}^{n}+y_{i-1, j-1}^{n}}{\Delta x \Delta v} \frac{y_{i+1, j}^{n}-y_{i, j}^{n}}{\Delta x}\left(\frac{y_{i, j+1}^{n}-y_{i, j}^{n}}{\Delta v}\right)^{2}\right\}+u_{i, j}^{n-1} \frac{y_{i+1, j}^{n}-y_{i-1, j}^{n}}{2 \Delta x}-v_{i, j}^{n-1}
\end{aligned}
$$

\section{Test problems}

Problem 1. The exact solutions of non-linear Burgers' equations (1) and (2) can be generated by using by the Hope-Cole transformation [5] which are

$$
\begin{aligned}
& y(x, u, t)=\frac{3}{4}-\frac{1}{4\left\{1+\exp \left((-4 x+4 u-t) \frac{R}{32}\right)\right\}} \\
& y(x, y, t)=\frac{3}{4}+\frac{1}{4\left\{1+\exp \left((-4 x+4 v-t) \frac{R}{32}\right)\right\}}
\end{aligned}
$$

The initial and boundary conditions are taken from exact solution and computational domain for this problem is,

$$
D=\{(x, u),(x, v): 0 \leq x \leq 1,0 \leq u, v \leq 1\}
$$

The numerical computations are performed using uniform grid, with a mesh widh $\Delta x=\Delta u=\Delta v=0.05$.

Problem 2. In this problem, the computational domain is taken as $D=\{(x, u),(x, v): 0 \leq x \leq 0.5,0 \leq u, v \leq 0.5\}$ and Burgers' equations (1), (2) are taken as with the initial conditions,

$$
\left.\begin{array}{l}
y(x, u, 0)=\sin \pi x+\cos \pi u \\
y(x, v, 0)=x+v
\end{array}\right\} 0 \leq x \leq 0.5,0 \leq u, v \leq 0.5
$$

and boundary conditions,

$$
\begin{gathered}
y(0, u, t)=\cos \pi u, y(0.5, u, t)=1+\cos \pi u \\
y(0, v, t)=v, \quad y(0.5, v, t)=0.5+v \\
0 \leq u, v \leq 0.5, t \geq 0
\end{gathered}
$$

\section{Numerical results and conclusions}

The variable interchange method for two-dimensional nonlinear Burgers' equations has been presented. The efficiency and numerical accuracy of the present method are validated trough two numerical examples. The accuracy of the numerical solutions indicates that the method is well suited for the solution of two-dimensional non-linear Burges' equation.

The results is given in Tables 1,2,3, and 4 at some typical mesh points demonstrate that the present method achieves similar results as those of Jain and Holla [3], A.R. Bahadır [6], Srivastaval et all. [9]. 
Table 1: Comparison of numerical values of $u$ for $R=500$ at $t=0.625$.

\begin{tabular}{|l|l|l|l|l|}
\hline \multirow{2}{*}{$(x, y)$} & \multicolumn{5}{|c|}{ Numerical values of $u$} \\
\cline { 2 - 5 } & \multicolumn{1}{|c|}{ Present work } & \multicolumn{1}{|c|}{ Bahadır [2] } & Jain and Holla [3] & Srivastava et al. [4] \\
\cline { 2 - 5 } & $\mathrm{N}=20$ & $\mathrm{~N}=20$ & $\mathrm{~N}=20$ & $\mathrm{~N}=20$ \\
\hline$(0.15,0.1)$ & 0.96652 & 0.96650 & 0.95691 & 0.96870 \\
\hline$(0.3,0.1)$ & 1.02972 & 1.02970 & 0.95616 & 1.03200 \\
\hline$(0.1,0.2)$ & 0.84451 & 0.84449 & 0.84257 & 0.86178 \\
\hline$(0.2,0.2)$ & 0.87633 & 0.87631 & 0.86399 & 0.86178 \\
\hline$(0.1,0.3)$ & 0.67811 & 0.67809 & 0.67667 & 0.67920 \\
\hline$(0.3,0.3)$ & 0.79793 & 0.79792 & 0.76876 & 0.79947 \\
\hline$(0.15,0.4)$ & 0.54603 & 0.54601 & 0.54408 & 0.66036 \\
\hline$(0.2,0.4)$ & 0.58877 & 0.58874 & 0.58778 & 0.58959 \\
\hline
\end{tabular}

Table 2: Comparison of numerical values of $v$ for $R=500$ at $t=0.625$.

\begin{tabular}{|l|l|l|l|l|}
\hline \multirow{2}{*}{$(x, y)$} & \multicolumn{5}{|c|}{ Numerical values of $v$} \\
\cline { 2 - 5 } & \multicolumn{1}{|c|}{ Present work } & \multicolumn{1}{|c|}{ Bahadır [2] } & Jain and Holla [3] & Srivastava et al. [4] \\
\cline { 2 - 5 } & $\mathrm{N}=20$ & $\mathrm{~N}=20$ & $\mathrm{~N}=20$ & $\mathrm{~N}=20$ \\
\hline$(0.15,0.1)$ & 0.09022 & 0.09020 & 0.10177 & 0.09043 \\
\hline$(0.3,0.1)$ & 0.10693 & 0.10690 & 0.13287 & 0.10727 \\
\hline$(0.1,0.2)$ & 0.17974 & 0.17972 & 0.18503 & 0.17295 \\
\hline$(0.2,0.2)$ & 0.16780 & 0.16777 & 0.18169 & 0.16816 \\
\hline$(0.1,0.3)$ & 0.26227 & 0.26222 & 0.26560 & 0.26268 \\
\hline$(0.3,0.3)$ & 0.23498 & 0.23497 & 0.25142 & 0.23550 \\
\hline$(0.15,0.4)$ & 0.31755 & 0.31753 & 0.32084 & 0.29019 \\
\hline$(0.2,0.4)$ & 0.30372 & 0.30371 & 0.30927 & 0.30419 \\
\hline
\end{tabular}

Table 3: Comparison of numerical values of $u$ for $R=50$ at $t=0.625$.

\begin{tabular}{|l|l|l|l|l|}
\hline \multirow{2}{*}{$(x, y)$} & \multicolumn{4}{|c|}{ Numerical values of $u$} \\
\cline { 2 - 5 } & \multicolumn{1}{|c|}{ Present work } & \multicolumn{1}{|c|}{ Bahadır [2] } & Jain and Holla [3] & Srivastava et al. [4] \\
\cline { 2 - 5 } & $\mathrm{N}=20$ & $\mathrm{~N}=20$ & $\mathrm{~N}=20$ & $\mathrm{~N}=20$ \\
\hline$(0.15,0.1)$ & 0.96690 & 0.96668 & 0.97258 & 0.97146 \\
\hline$(0.3,0.1)$ & 1.14830 & 1.14827 & 1.16214 & 1.15280 \\
\hline$(0.1,0.2)$ & 0.85912 & 0.85911 & 0.86281 & 0.86307 \\
\hline$(0.2,0.2)$ & 0.97639 & 0.97637 & 0.96483 & 0.97981 \\
\hline$(0.1,0.3)$ & 0.66020 & 0.66019 & 0.66318 & 0.66316 \\
\hline$(0.3,0.3)$ & 0.76933 & 0.76932 & 0.77030 & 0.77230 \\
\hline$(0.15,0.4)$ & 0.57969 & 0.57966 & 0.58070 & 0.58180 \\
\hline$(0.2,0.4)$ & 0.75680 & 0.75678 & 0.74435 & 0.75856 \\
\hline
\end{tabular}


Table 4: Comparison of numerical values of $v$ for $R=50$ at 0.625 .

\begin{tabular}{|l|l|l|l|l|}
\hline \multirow{2}{*}{$(x, y)$} & \multicolumn{4}{|c|}{ Numerical values of $v$} \\
\cline { 2 - 5 } & Present work & \multicolumn{1}{|c|}{ Bahadır [2] } & Jain and Holla [3] & Srivastava et al. [4] \\
\cline { 2 - 5 } & $\mathrm{N}=20$ & $\mathrm{~N}=20$ & $\mathrm{~N}=20$ & $\mathrm{~N}=20$ \\
\hline$(0.15,0.1)$ & 0.09826 & 0.09824 & 0.09773 & 0.09869 \\
\hline$(0.3,0.1)$ & 0.14113 & 0.14112 & 0.14039 & 0.14158 \\
\hline$(0.1,0.2)$ & 0.16683 & 0.16681 & 0.16660 & 0.16754 \\
\hline$(0.2,0.2)$ & 0.17067 & 0.17065 & 0.17397 & 0.17110 \\
\hline$(0.1,0.3)$ & 0.26262 & 0.26261 & 0.26940 & 0.26378 \\
\hline$(0.3,0.3)$ & 0.22579 & 0.22576 & 0.22463 & 0.22654 \\
\hline$(0.15,0.4)$ & 0.32747 & 0.32745 & 0.32402 & 0.32851 \\
\hline$(0.2,0.4)$ & 0.32443 & 0.32441 & 0.31822 & 0.32500 \\
\hline
\end{tabular}

\section{Competing interests}

The authors declare that they have no competing interests.

\section{Authors' contributions}

All authors have contributed to all parts of the article. All authors read and approved the final manuscript.

\section{References}

[1] J.D. Boadway, Transformation of elliptic partial differential equations for solving two-dimensional boundary problems in fluid flow, Int. Numer. Meth. Engng. 10 (1976) 527.

[2] C.A.J. Fletcher, Generating exact solutions of two-dimensional Burgers' equation, Int. J. Numer. Meth. Fluids 3 (1983) $213-216$.

[3] P.C. Jain, D.N. Holla, Numerical solution of coupled Burgers' equations, Int. J. Numer. Meth. Eng. 12 (1978)213-222.

[4] O. Goyon, Multilevel schemes for solving unsteady equations, Int. J. Numer. Meth. Fluids 22 (1996) 937-959.

[5] F.W. Wubs, E.D. de Goede, An explicit-implicit method for class of time-dependent partial differential equations, App. Numer. Math. 9 (1992) 157-181.

[6] A.R. Bahadır, A fully implicit finite-difference scheme for two-dimensional Burgers' equations, Applied Mathematics and Computataion, 137 (2003) 131-137.

[7] S.M. El-Sayed, D. Kaya, On the numerical solution of the system of two-dimensional Burgers' equations by the decomposition method, Applied mathematics and Computation, 158 (2004) 101-109.

[8] H. Zhu, H. Shu, M. Ding, Numerical solutions of two-dimensional Burgers' equations by discrete Adomian decomposition method, Computers and Mathematics with Applications, 60 (2010) 840-848.

[9] V.K. Srivastava, M. Tamsir, U. Bhardwaj, Y. Sanyasiraju, Crank-Nicolson scheme for numerical solutions of two-dimensional coupled Burges' equations, International Journal of Scientific and Engineering Research, 2(2011) 1-7.

[10] V. Gülkaç, Numerical solution of two-dimensional Schrödinger equation by Boadway transformation, International Journal of Computer Mathematics, 80 (2003) 1543-1548.

[11] T. Öziş, V. Gülkaç, Application of variable interchange method for solution of two-dimensional fusion problem with convective boundary conditions, Numerical Heat Transfer, Part A, 44 (2008) 85-95.

[12] V. Gülkaç, T. Öziş, Treatment of two-dimensional moving boundary problem by Boadway's transformation, Bull. Cal. Math. Soc. 88 (1996) 253-260. 